\title{
Visual cues of portion sizes: the role of conservation
}

\author{
M. A. Defeyter \\ Department of Psychology, Northumbria University, UK
}

\begin{abstract}
Young children have the ability to regulate intake both within meals and throughout the course of the day. However, by the age of 5 years, children's ability to self-regulate energy is subject to modification by social and environmental factors. The present study examines the influence of 5 to 6-year-olds failure to conserve volume on their perceptions of portion size, hunger and satiety. This experiment was a between subjects design with one factor and two levels (wide and short cereal bowl $v$. narrow and tall cereal bowl). Children were pseudorandomly allocated to conditions according to age and BMI. Forty participants (45\% male), with a mean age of 5.6 years (range: 5-6.3 years) were recruited from schools providing breakfast in the North East of England. Children were individually tested on a Piagetian conservation of volume task by pouring a single-sized portion of cereal (Rice Krispies) into two same-sized bowls. All children agreed that the bowls contained the same amount of cereal. The experimenter then poured the cereal from one bowl into a taller yet narrower bowl. At test, all children stated that the taller bowl contained a larger portion of cereal. Half of the participants were then given the cereal in the tall bowl to eat, and the remaining children were given the cereal in the short bowl to eat accompanied by $125 \mathrm{ml}$ of semi-skimmed milk. Thirty minutes following breakfast consumption, children were asked satiety questions using semantic differential scales. Despite having consumed exactly the same amount of cereal, children in the taller bowl condition perceived themselves as being more sated than those in the short bowl condition $(t(38)=2.56, P<0.05)$. There was also a significant effect of bowl size on hunger, with children in the tall bowl condition reporting that they were significantly less hungry than children in the short bowl condition $(t(38)=3.12, P<0.01)$. There was no significant effect of condition on thirst $(t(38)=1.96, P>0.05)$. These results suggest that visual cues may play an important role in determining portion size and perceptions of hunger and satiety.
\end{abstract}

